\title{
La arqueología de la guerra y el surgimiento de sociedades complejas en los llanos del Orinoco
}

The Archaeology of Warfare and the Emergence of Complex Societies in the Llanos of the Orinoco

\author{
Juan Carlos Vargas Ruiz \\ Universidad de Pittsburgh, Estados Unidos \\ jcv12@pitt.edu
}

\begin{abstract}
RESUMEN
El presente artículo discute el papel que desempeñó la guerra en el surgimiento de una jerarquización social en los llanos del Orinoco, en Colombia y Venezuela. Se presenta una discusión en torno a los modelos etnohistóricos y arqueológicos más relevantes relacionados con el área en la que se sintetizan sus principales elementos, y se propone una estrategia metodológica para su evaluación arqueológica. Como conclusión, se argumenta que, aunque la guerra estuvo presente en los Ilanos de Venezuela, las tendencias tempranas a la centralización estuvieron relacionadas más con factores agrícolas que bélicos.
\end{abstract}

Palabras clave: arqueología, llanos del Orinoco, guerra. $\overline{\text { ABSTRACT }}$

This paper discusses the role played by warfare in the emergence of social hierarchization in the Savannas of the Orinoco in Colombia and Venezuela. Here the main elements of the ethnohistorical and archaeological models proposed for the region are summarized. In addition a methodological strategy to the archaeological analysis of warfare in the Llanos is presented. In conclusion it is argued that although warfare was present in the Llanos of Venezuela, the earliest trends to demographic centralization were related to agriculture rather than military factors.

Keywords: Archaeology, Savannas of the Orinoco, warfare. 


\section{Introducción ${ }^{1}$}

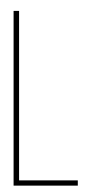

as fuentes etnohistóricas han inspirado diferentes ideas acerca del papel de la guerra en el surgimiento de las sociedades complejas en los llanos de Colombia y Venezuela. Algunos autores le atribuyen un papel central (Redmond, Gassón y Spencer 1999; Spencer 1998); otros, aunque consideran que la guerra estuvo presente en la región, plantean que no fue una fuerza fundamental para el surgimiento de jerarquías sociales (Gassón 2009). Hasta el momento, los modelos arqueológicos relativos a los llanos del Orinoco se han centrado en discutir las causas por las que la guerra estuvo presente en tiempos prehispánicos, y las condiciones en las que se desarrolló, para lo cual la información etnohistórica ha sido bastante útil al momento de ilustrar los argumentos contenidos en dichos modelos. Sin embargo, es poco lo que se ha avanzado en la evaluación arqueológica de dicha información. El presente artículo propone estrategias metodológicas que permiten la evaluación de los modelos etnohistóricos y arqueológicos que consideran la guerra como la principal causa de complejización social en esta macrorregión.

\section{El modelo etnohistórico}

Sin duda alguna, las características ecológicas de los llanos del Orinoco han sido determinantes en la construcción de los modelos etnohistóricos y arqueológicos que dan cuenta de la organización sociopolítica y económica de los grupos que habitaron esta región en el pasado (Gassón 1998; Morey 1975; Spencer 1994).

El trabajo más importante e influyente sobre la etnohistoria de los llanos del Orinoco es, sin duda, el de Nancy Morey (1975). Esta antropóloga, con base en un estudio minucioso de las fuentes documentales, llegó a la conclusión de que, en los llanos de Colombia y Venezuela, existieron sociedades complejas mucho

1 La idea inicial de este artículo surgió al finalizar la tesis de maestría. Una versión en borrador fue presentada en el marco de un curso seminario titulado "Archaeology of Warfare", realizado durante el doctorado. La presente versión incluye nuevos elementos que el autor ha desarrollado desde entonces. 
antes de la invasión europea y que el surgimiento de la complejidad social en la región se relacionó con la guerra ${ }^{2}$, la cual estuvo determinada por aspectos ecológicos. Otro de los trabajos que abordó tempranamente la relación entre guerra, ecología, economía y complejización social fue desarrollado por Morey y Marwitt (1975), quienes afirmaron, al igual que Morey (1975), que en las terrazas aluviales de los llanos la guerra con objetivos económicos, como la expansión territorial y la tributación por parte de los grupos políticamente centralizados, fue una respuesta a la presión poblacional y la limitación de recursos, especialmente de tierras aptas para el cultivo.

La hipótesis ecológica sobre la guerra se sustenta en información etnohistórica que sugiere que algunos grupos, como los caquetíos, los guayupes, los salivas y los achaguas, reconocidos por los cronistas por su belicosidad y complejidad, al parecer controlaban grandes extensiones de tierra apta para la agricultura y luchaban por expandir su territorio (Federmann 1958; Morey 1975; Morey y Marwitt 1975; Rivero 1956). Estos grupos ocupaban principalmente áreas de bosques a lo largo de los afluentes de ríos como el Casanare, el Meta, el Arauca, el Apure y el Anaro, donde abundan suelos fértiles y se concentran importantes cantidades de especies animales. En general, las poblaciones en esta región evitaban asentarse en las terrazas aluviales primarias para prevenir las inundaciones anuales; tampoco ocupaban las sabanas por su baja productividad agrícola y la alta impredictibilidad en la consecución de otros recursos alimenticios (Morey 1975).

Las crónicas mencionan que los guayupes, caquetíos y achaguas vivían en poblados nucleados, compuestos por entre doscientas y trescientas personas, las cuales tenían su propio jefe. En algunas zonas existía un cacique que vivía en un poblado central más grande y que ejercía su autoridad sobre un grupo de líderes locales que vivían en asentamientos cercanos de menor tamaño (Morey 1975; Rivero 1956). Esta distribución era ventajosa en términos de la defensa de los sitios, en cuanto permitía la movilización y coordinación de guerreros ante algún ataque (Federmann 1958), a la vez que posibilitaba agrupar la suficiente fuerza de trabajo para ser invertida en los campos de cultivo comunales, localizados en la periferia de los asentamientos (Carvajal 1985; Morey 1975; Rivero 1956).

En los llanos, algunos grupos vivieron en asentamientos fortificados, en áreas donde la defensa fue crítica para la supervivencia (Castellanos 1962; Federmann 1958; Morey 1975; Rivero 1956). La existencia de fortificaciones es un claro indicador de guerra en cualquier región (Allen 2006; Arkush y Stanish 2005; Keeley, Fontana y Quick 2007; Lau 2010). Estas estructuras prestaban protección a

2 En este artículo entendemos guerra como la acción grupal organizada y voluntaria en contra de otro grupo, que involucra el uso potencial o real de fuerza letal (Ferguson 1990). 
la población que habitaba en su interior y a la vez eran un elemento de disuasión para los enemigos (Roscoe 1998); también podían servir como una forma de control sobre el grupo local (Allen 2006). Cerca al alto río Meta, al parecer, existió un poblado achagua en el cual, según los cronistas, había alrededor de 100 viviendas multifamiliares (de 800 a 1.000 personas) (Aguado 1950; Rivero 1956). Este poblado de los catarubeni estaba rodeado de una estructura en tierra sobre la cual se dispuso una empalizada. Los reportes indican que este tipo de poblados también existieron en el curso de los ríos Cusiana, Pauto, Ariari, Apure, Sarare y en el alto Orinoco. Rivero (1956) sugiere que estos poblados estaban separados por una distancia de entre 7 y 8 kilómetros, lo que indica la posible existencia de "zonas de amortiguamiento" (buffer zones) entre las distintas comunidades. Las zonas de amortiguamiento han sido asociadas con áreas con densidad demográfica alta y con concentración diferencial de recursos, como la cuenca del Mississippi, Mesa Verde, Nueva Guinea y Mesoamérica, entre otras (Allen 2008; Anderson 1994; Dye 2006; LeBlanc 2006).

Las crónicas también describen la existencia de pequeños caseríos (de entre 20 y 30 personas), dispersos a través de las unidades de bosque y bancos, los cuales estaban retirados entre sí por entre 3 y 4 kilómetros. Los jiraras, los otomacos y los betoys fueron algunos de los grupos que implementaron este patrón de asentamiento (Rivero 1956). Esta distribución espacial pudo ofrecer una oportunidad a los pobladores antiguos para escapar a alguna zona de refugio en caso de algún ataque o emboscada.

Con base en estas características de los patrones de asentamiento, surge la pregunta sobre si la aparición de poblados fortificados fue una consecuencia de la penetración europea o si el patrón extendido entre las comunidades indígenas prehispánicas de los llanos del Orinoco se caracterizó por pequeños caseríos dispersos. Morey (1975) y Morey y Marwitt (1975) afirman que el poblado fortificado no fue el patrón de asentamiento preferido en los llanos antes de la invasión europea, y que fue implementado solo en regiones con altos niveles de violencia intergrupal causada por disputas sobre tierras para cultivo. Es probable que la popularización de poblados fortificados haya sido una consecuencia de la expansión europea, la cual produjo un incremento en la frecuencia de violencia intergrupal en los llanos. Por ejemplo, grupos como los caribes y los guahibos muy tempranamente se aliaron con los europeos y se dedicaron a capturar esclavos para su comercialización (Whitehead 1994, 1998). Sin embargo, parece que la caza de esclavos fue una práctica extendida entre algunos grupos llaneros como los achaguas y los caquetíos antes de la penetración europea. Algunos cronistas plantean que el principal motivo de enfrentamiento entre las 
comunidades llaneras fue la captura de mujeres y niños como fuerza de trabajo agrícola (Federmann 1958; Gumilla 1963; Rivero 1956), con el objetivo de aumentar la producción de excedentes para las élites locales y regionales. Adicionalmente, la posesión de esclavos, al parecer, era usada como una demostración de poder, riqueza y autoridad entre las élites llaneras.

Es probable que, antes de la invasión europea, la guerra y el comercio en el Orinoco hayan sido determinados por principios étnicos (Heckenberger 2002). Los grupos arawaks, como los achaguas o los caquetíos, establecieron redes de intercambio con grupos relacionados culturalmente, por lo que desarrollaron fuertes prohibiciones ideológicas y sociales en contra de la guerra con otros arawaks. De acuerdo con los datos etnohistóricos, los grupos esclavizados eran reconocidos como culturalmente inferiores y se usaba la denominación mako para diferenciarlos; esta misma nominación se usó para referirse a los grupos de cazadores-recolectores de sabana (Morey 1975). De esta forma, una de las consecuencias de la especialización productiva ecológica en el Orinoco pudo ser que los límites étnicos también fueron económicos (Whitehead 1994).

Sin embargo, existen crónicas que indican que el conflicto violento en el interior del grupo étnico fue posible, a pesar de los argumentos que sugieren la prohibición de la guerra entre grupos arawaks ${ }^{3}$. Por ejemplo, hay reportes que sugieren que los achaguas estaban en continua guerra entre ellos mismos a la llegada de los españoles, lo que había reducido significativamente su población (Rivero 1956). De ser así, es muy probable que la competencia entre facciones derivara en violencia entre comunidades de una misma unidad política, cuando los conflictos no podían ser resueltos por medios pacíficos. Una de las consecuencias de la expansión de la autoridad cacical hacia el exterior de la comunidad pudo ser el incremento de confrontaciones a medida que los líderes regionales se distanciaban de los jefes de rango menor (Kolb y Dixon 2002).

Finalmente, existe la posibilidad de que el contexto histórico descrito por las fuentes documentales corresponda exclusivamente al periodo de la Colonia (Ferguson 1990). Gassón (2009) ha planteado que, a pesar de la abundante información etnohistórica que se refiere a la guerra y a la violencia intergrupal, las crónicas están separadas por más de cinco siglos de las fechas reportadas en aquellos sitios arqueológicos que han sido registrados en los llanos, y que estas descripciones pueden ser el resultado de los cambios sociopolíticos introducidos por los invasores europeos y también por un medioambiente significativamente diferente. En contraste, Morey (1975) afirma que hay reportes tempranos que 
indican la belicosidad de las poblaciones arawaks y los problemas que los españoles confrontaron al conquistar los llanos durante los primeros años del siglo XVII. En su opinión, es más probable que los arawaks hayan sido afectados muy rápidamente por epidemias transmitidas por los europeos y también por su expansión militar.

En resumen, la información etnohistórica que he presentado y discutido permite generar las siguientes hipótesis en relación con la guerra en los llanos:

1. El surgimiento de las sociedades complejas sucedió siglos antes y sin la presencia de invasores europeos.

2. La guerra fue un fenómeno que estuvo presente desde mucho antes de la invasión española.

3. La guerra fue motivada por escasez de tierras aptas para la agricultura como efecto del crecimiento demográfico y la presión poblacional.

4. La nucleación de la población fue una consecuencia de la guerra.

5. El factor crítico en los llanos no fue la tierra sino la fuerza de trabajo.

\section{Los modelos arqueológicos}

Los estudios arqueológicos con una perspectiva regional y sistemática sobre los llanos del Orinoco han sido pocos y han estado concentrados en Barinas, Venezuela. El primer reconocimiento regional sistemático fue adelantado por Spencer y Redmond (1992) en la década de 1980, en la región de Gaván, y abarcó un área de más de $400 \mathrm{~km}^{2}$. El segundo estudio fue realizado por Gassón (1998) en la década de 1990, en la región de Cedral, y abarcó 60 km². En estas dos regiones, durante el periodo Gaván Tardío (A. D. 550-1000), surgieron dos unidades políticas regionales presuntamente caquetías (arawaks).

En la región de Gaván se ha registrado una jerarquía regional de asentamientos compuesta por tres niveles. El primer nivel de dicha jerarquía se ha asignado al sitio El Gaván, el cual tiene una extensión de 35 ha, exhibe montículos de gran tamaño alrededor de una plaza central y un encerramiento hecho en tierra sobre el que se instaló una empalizada. Desde El Gaván parten calzadas monumentales hacia sitios de menor tamaño (5 ha-10 ha), en donde no existen encerramientos ni otras estructuras defensivas, pero en los que se observan de uno a tres montículos de tamaño considerable. Al tercer nivel han sido asignados sitios dispersos bastante pequeños ( 0,5 ha-2 ha) que carecen de arquitectura monumental. Spencer (1998) propone, basado en estas características de los 
patrones de asentamiento, el surgimiento de una organización política cacical regional, centralizada y no burocrática durante el periodo Gaván Tardío. La existencia de campos elevados en uno de los centros secundarios evidencia que la agricultura fue intensificada y los excedentes producidos, movilizados hacia el centro regional. La presencia de artefactos exóticos provenientes de los Andes y otras regiones distantes sugiere que el intercambio a larga distancia fue importante para los líderes regionales (Spencer y Redmond 1992). Algunas diferencias en los patrones de enterramiento, los ajuares funerarios y el tamaño y los conjuntos de artefactos asociados a las unidades domésticas en el centro El Gaván indicarían una marcada diferenciación social (Spencer 1998).

Durante el periodo Gaván Tardío, en la región vecina de Cedral surgió otra unidad política que comparte los principales rasgos con el cacicazgo de Gaván: jerarquía de asentamientos compuesta por al menos tres niveles de toma de decisión, monumentalidad, intensificación de la agricultura en campos levados, intercambio a larga distancia y estructuras defensivas como encerramientos y empalizadas, y también calzadas (Gassón 1998). En Cedral tampoco existe evidencia de que la población regional haya excedido el potencial productivo (Gassón 1998). A pesar de estas semejanzas, es posible observar significativas diferencias entre el registro arqueológico de Cedral y Gaván (Gassón y Rey 2006). Primero, el centro primario regional del Cedral (135 ha) excede 4 veces en tamaño al de El Gaván (35 ha). El número de centros secundarios en Cedral es mayor que en Gaván y algunos de ellos equivalen e incluso exceden en tamaño al centro primario de El Gaván. Tercero, los campos elevados del Cedral están asociados directamente al centro primario, mientras que en Gaván se encuentran próximos a un centro secundario. Cuarto, la red de calzadas que une el centro primario con los secundarios en el Cedral es mucho más extensa que la de El Gaván.

Con base en estas líneas de evidencia, Redmond, Gassón y Spencer (1999) han caracterizado las sociedades complejas de El Cedral y El Gaván como cacicazgos cíclicos. El proceso cíclico se refiere a

las transformaciones que ocurren cuando los niveles administrativos y de toma de decisiones dentro de las sociedades cacicales que ocupan una región fluctúan entre uno y dos niveles por encima de la comunidad local. Este es el proceso recurrente del surgimiento, expansión y fragmentación de los cacicazgos complejos en medio de un contexto regional de cacicazgos simples. (Anderson 1994, 9)

Es probable que este proceso de surgimiento y expansión en Barinas haya resultado en la aniquilación violenta del centro primario de El Gaván por medio 
de una conflagración, posiblemente causada por los habitantes del Cedral hacia el 1000 A. D. (Redmond, Gassón y Spencer 1999).

El modelo propuesto por Spencer (1998) contempla la guerra como un fenómeno regular y esencial para la consolidación e institucionalización del liderazgo, en cuanto permitió el desarrollo de las dimensiones interna y externa de la autoridad de los líderes emergentes (Spencer 1993, 1994). Este autor considera que la principal causa para el conflicto regional en la región de Gaván fue el crecimiento poblacional constante, y una pronunciada circunscripción ambiental y social causada por la escasez de tierras aptas para el cultivo (Spencer 1998). Aunque Spencer afirma que en esta región no hubo presión poblacional sobre los recursos, coincide con Morey y Marwitt (1975) en que la circunscripción ambiental determinó la configuración de un contexto social en el que la competencia cada vez fue más violenta (Spencer 1998). En contraste, hay algunos investigadores que argumentan que la causa de la guerra en los llanos del Orinoco fue la captura de fuerza de trabajo, más que la expansión territorial (Drennan 1995; Gassón 1998; Whitehead 1994). De acuerdo con estos investigadores, el factor limitante para la producción agrícola en los llanos de la Orinoquia no fueron las tierras para cultivo, sino la mano de obra. En este sentido, Whitehead (1994) plantea que el control de las personas, más que la expropiación o redistribución de recursos económicos específicos, fue la máxima expresión de dominancia en el Orinoco.

En contraste, el modelo propuesto por Gassón (2009) implica el argumento de que aunque la guerra estuvo presente en Barinas, y por extensión en el Orinoco, no fue un fenómeno regular y endémico y, por el contrario, sugiere que los líderes de estas unidades políticas quizá hicieron mayor énfasis en aspectos ceremoniales e ideológicos para legitimar e institucionalizar su liderazgo. Gassón (2009) critica el modelo propuesto por Spencer por lo que llama excesiva influencia mesoamericana, y opina que el énfasis en la guerra como un factor central para el surgimiento de organización social compleja no corresponde a la realidad de los grupos llaneros prehispánicos. Este investigador también opina que las evidencias que Spencer presenta para apoyar su argumento en favor de la guerra no son concluyentes o resultan insuficientes. Por ejemplo, aunque se plantea que el centro primario El Gaván fue destruido por una conflagración durante un ataque del Cedral (Redmond, Gassón y Spencer 1999), dicho suceso quizá pudo estar relacionado con una intensa sequía que se presentó hacia el 800-900 A. D., la cual ha sido registrada en la cuenca del Cariaco (Gassón y Zucchi 2010). Gassón (2009) también propone que muchas de las estructuras monumentales que, según Spencer y Redmond $(1992,1998)$, sirvieron para la defensa de los sitios, como los encerramientos ovalados, podrían haber sido construidas de acuerdo con concepciones ecológicas e ideológicas arawaks. 
Con base en la información de los dos reconocimientos regionales se puede concluir que:

1. El surgimiento de una organización social compleja ocurrió mucho antes de la llegada de los españoles.

2. Indicadores arqueológicos como encerramientos y zonas de amortiguamiento sugieren que la guerra fue un fenómeno presente en Barinas durante el periodo Gaván Tardío. Estos indicadores pueden ser interpretados desde una lógica diferente, relacionada con factores ideológicos y ecológicos.

3. Pese a las evidencias arqueológicas, no es claro cuál fue la causa de la guerra en la región. Spencer (1998) plantea que fue motivada por circunscripción social y ambiental producida por la escasez de tierras para cultivo. Por su parte, Whitehead (1994), Drennan (1995) y Gassón (1998) piensan que la principal causa fue la escasez de mano de obra.

4. Aunque se reconoce que en la región hubo guerra, no hay un acuerdo sobre la importancia que tuvo este fenómeno para el surgimiento de la organización social compleja en la región.

5. No es claro si la nucleación de población fue causada por la guerra o si otros factores pudieron incidir en la configuración de este patrón de distribución demográfica.

6. Las demás evidencias circunstanciales que se han presentado son refutables.

Ya que ninguno de los dos modelos ha contado con evidencia sólida que lo soporte, a continuación presento una propuesta que, considero, podría ser útil para evaluar la guerra en los llanos. Esta propuesta se basa en los datos provenientes de los reconocimientos efectuados por Spencer y Redmond (1992), Gassón (1998) y en mis propios datos de campo (Vargas 2011).

\section{Estrategia metodológica para la evaluación arqueológica de la guerra en los llanos}

La evaluación de las hipótesis derivadas de la información etnohistórica y arqueológica en relación con la guerra en los llanos requiere incluir análisis arqueológicos que aporten nueva información. Para esto he evaluado las siguientes variables. 


\section{Agregación de población}

Uno de los efectos de la guerra es la agregación de la población con el objetivo de reducir el riesgo (Allen 2008; Dye 2006; Earle 1997; Junker 1999; LeBlanc 2006). La proximidad entre los distintos miembros y familias pudo permitir disponer de la suficiente fuerza para la defensa de los sitios. Un método que puede ser útil para medir la agregación de población es el análisis de vecino más cercano (nearest neighbor analysis) ${ }^{4}$. El análisis del vecino más cercano permite observar si la distribución espacial de asentamientos en una región tiende a la agregación o a la dispersión. Para usar la metodología de vecino más cercano es importante considerar los límites de la región por analizar, ya que de ellos dependerán los valores de índice obtenidos.

Es importante advertir que, aunque en las regiones de Gaván y Cedral los asentamientos estuvieron localizados a lo largo de los caños y ríos secundarios, estos son de difícil navegabilidad porque son angostos y permanecen con niveles bajos, por lo que es bastante posible que la mayoría de desplazamientos se hicieran a pie, al menos durante una gran parte del año. Quizá esta sea una de las razones que expliquen por qué la gente construyó calzadas para comunicar los asentamientos en Barinas. Lo anterior no es aplicable a otras regiones de los llanos, donde las condiciones de navegabilidad pudieron ser mejores, por lo que el uso de este análisis debe atender a esa particularidad.

En este análisis se ha decidido considerar que una persona podía recorrer más o menos 12 kilómetros en cualquier dirección durante un día de camino. De manera que esta distancia radial es la que he asumido como el límite de las unidades políticas de Gaván y Cedral. También es importante tener en cuenta que la gente en ambas regiones no vivía a lo largo del mismo caño o río secundario y, por tanto, la agregación no es simplemente un efecto de una distribución afectada por este factor. Finalmente, es preciso entender que los valores de vecino más cercano sencillamente permiten cuantificar y cualificar una característica de la distribución de la población que ha sido relacionada con la guerra, la agregación, que debe ser correlacionada con otras líneas de evidencia.

4 El coeficiente de vecino más cercano (R) es definido como el radio promedio sobre todos los puntos de distancias entre un punto y el otro punto más cercano a este, dividido entre la distancia promedio esperada si el mismo número de objetos estuviese distribuido en forma aleatoria sobre la misma área (Kintigh 1990). Una distribución aleatoria de puntos produce un valor de vecino más cercano próximo a 1,00; una distribución nucleada produce un valor menor a 1,00 por encima de 0, y una distribución en la que los objetos están más dispersos exhibirá valores mayores a 1,00 y hasta 2,15, que es el límite (Kintigh 1990; Pinder, Shimada y Gregory 1979). 
El análisis intrarregional de la unidad política de Gaván indica que los asentamientos tendieron a la agregación $(R=0,915, p>0,4)$; aunque los sitios de tercer nivel estuvieron dispersos, estos permanecieron próximos al centro primario, como indica la distancia observada $(r o=1.210 \mathrm{~m}$ ). Las proyecciones de población realizadas por Spencer y Redmond muestran que el $30 \%$ de la población regional vivió concentrada en el centro regional.

En el Cedral, el $40 \%$ de la población regional estuvo concentrada en el centro primario regional, mientras los centros secundarios se ubicaron a considerable distancia de este y los sitios de tercer nivel lo hicieron alrededor. Un problema de esta región es que se tiene muy poca información sobre los sitios de tercer nivel, ya que el reconocimiento regional solo estuvo dirigido a identificar los sitios monumentales (Gassón 1998). De esta manera, los valores observados sobre la distancia con respecto al vecino más cercano podrían variar en la medida en que se recupere información sobre esta clase de sitios. En esta región, los límites para el análisis se definieron en dos formas. Primero, se tomó la distancia radial de $12 \mathrm{~km}$ y se analizaron los sitios incluidos en esta. Segundo, se tomaron todos los sitios conectados al centro regional el Cedral por medio de la red de calzadas. En el primer caso, el índice $(\mathrm{R}=1.605,0,01>p>0,005)$ sugiere que los asentamientos en el área de estudio tendieron a estar dispersos $(r o=2.273,9 \mathrm{~m} ; r e=1.416,7 \mathrm{~m})$. Este puede ser un efecto del sesgo introducido en la muestra en cuanto no se incluyen todos los sitios pertenecientes a la unidad política. En el segundo caso, que corresponde a un área mucho más amplia definida a partir de la red de calzadas que conectan todos los sitios monumentales en la región, el índice de vecino más cercano $(\mathrm{R}=0,57, p>0,005)$ sugiere una desviación de una distribución aleatoria más fuerte y una tendencia intrarregional a la agregación entre asentamientos ( $r o=3.823,8 \mathrm{~m}$; $r e=6.638,9 \mathrm{~m}$ ).

Al unir las regiones de Gaván y Cedral, el índice de vecino más cercano ( $\mathrm{R}=0,403,0,01>p>0,005)$ sugiere que a escala macrorregional hubo una tendencia a la agregación de asentamientos.

En conclusión, los resultados del análisis de vecino más cercano indican que los distintos asentamientos, en las regiones de Gaván y Cedral, tendieron a la agregación. Esto confirmaría uno de los rasgos de los patrones de asentamiento sugeridos por los cronistas europeos, el cual es que las poblaciones indígenas llaneras prefirieron mantener sus poblados cerca unos de otros.

\section{Centralización demográfica}

Los principios de distancia-interacción hacen posible reconocer la organización regional centralizada, ya que los patrones de centralidad de interacción social 
tienden a atraer las poblaciones hacia un centro (Drennan y Peterson 2008). Las crónicas sugieren que, en los llanos, la gente se distribuyó alrededor de un lugar central, el cual dominaba otros asentamientos de menor tamaño que, aunque separados, estaban próximos al asentamiento principal. Este patrón de distribución de la población corresponde a un sistema de asentamiento centralizado demográficamente, en el cual la interacción constante y la reducida distancia entre los distintos asentamientos pudieron permitir la defensa eficiente de la unidad política. En un artículo anterior (Vargas 2012) he expuesto los análisis detallados de centralización demográfica en las regiones de Gaván y Cedral. Por esta razón, en este apartado solo presentaré las conclusiones de dichos análisis relevantes para la discusión sobre la guerra en los llanos.

Los análisis de centralización en las regiones de Gaván y Cedral sugieren que, a escala regional, el crecimiento demográfico estuvo acompañado por un incremento en las tendencias de centralización y un aumento en la integración política de la población hacia el periodo Gaván Tardío. Los análisis también muestran la reducción en los valores de centralización en el ámbito macrorregional (incluidos todos los asentamientos de las dos regiones), la cual pudo estar relacionada con el surgimiento de centros secundarios y procesos de fisión. Esta estrategia quizá permitió una amplia y eficiente distribución de la población a través del territorio cacical que obedecería a factores como la guerra entre unidades políticas (interregional) y no intrarregional, en cuanto en el interior de las regiones esas unidades mantuvieron su cohesión, como lo muestran los valores de centralización y la red de calzadas que comunicaba los sitios. Dicha distribución, en términos logísticos, probablemente permitió la rápida movilización y coordinación de fuerza para la defensa y el combate hacia el centro regional, pero también hacia otras zonas del territorio de la unidad política, tal como lo sugieren los cronistas europeos.

\section{Tamaño de las unidades políticas}

Peterson y Drennan (2005) han señalado que la definición de las escalas a las que las estructuras de interacción de la comunidad se configuran es fundamental para el estudio comparativo de las sociedades complejas. Por consiguiente, es importante, al momento de evaluar la guerra en una región, considerar la asimetría entre unidades políticas. Por ejemplo, una unidad política pequeña no estará capacitada para conquistar a una unidad política más grande, por lo que se espera que la última, eventualmente, conquiste a la primera (LeBlanc 2006). De igual forma, aunque una región sea más grande y posea más recursos, si se encuentra fragmentada en múltiples unidades políticas, hay mayor posibilidad de 
que sea conquistada por una unidad política que integre todas las comunidades en una región de menor extensión y con menores recursos disponibles (LeBlanc 2006). La capacidad que existe en cada una de las regiones para intensificar la producción también es importante a fin de determinar la asimetría entre unidades políticas, ya que, dependiendo de las diferencias en los potenciales de cada una, es más probable que aquellas unidades asentadas en regiones con mayor potencial de intensificación dominen a las de regiones con menor potencial. De esta forma, las diferencias en el tamaño de las unidades políticas y su capacidad para la intensificación económica permiten predecir qué grupo o grupos podrán vencer en caso de enfrentamiento (LeBlanc 2006).

Como hemos visto a propósito de los llanos de Barinas, los análisis de centralización sugieren que el tamaño de las unidades políticas, en algunos casos, como en la región de Cedral, quizá fue mayor que el estimado hasta ahora. Lo mismo sucede al considerar el tamaño de los asentamientos. Es bastante relevante reconocer que los sitios más grandes y densamente poblados pueden dominar territorios más amplios que los de menor tamaño (Peterson y Drennan 2005). Como se ha mencionado, hay diferencias notorias entre los centros primarios y secundarios de las regiones de Gaván y Cedral; también en las redes de calzadas que unen dichos sitios y la extensión de los sistemas de campos elevados para cultivo en cada región (Gassón y Rey 2006; Redmond, Gassón y Spencer 1999). Estas diferencias sugieren que, en el pasado, en los llanos existieron unidades políticas de diferente tamaño y, en consecuencia, diferentes niveles y grados de interacción social. De ser así, es probable que haya habido jefes poderosos, cuyos rangos y estatus dependían del número de comunidades que tenían bajo su control, lo que sería apoyado por las crónicas (Morey 1975).

La integración de unidades políticas del tamaño del Cedral podría estar relacionada con la hipotética guerra expansionista de los grupos caquetíos, reseñada en los documentos etnohistóricos (Morey 1975), grupos que probablemente dominaron a varias comunidades locales en distintas regiones, como una forma de garantizar su acceso a buenos suelos para cultivo y mano de obra. Como indiqué anteriormente, una de las hipótesis acerca del colapso de la unidad política de El Gaván sugiere la violenta aniquilación del centro primario de El Gaván a causa de un ataque infligido por habitantes del Cedral hacia el 1000 A. D. (Redmond, Gassón y Spencer 1999). De ser correcto el planteamiento de Redmond, Gassón y Spencer (1999), este sería un caso en el que una unidad política de gran tamaño, como la de Cedral, y con mayor capacidad para la intensificación agrícola, conquistó o eliminó a una de menor tamaño, como lo fue la de Gaván. Esta situación estaría acorde a la propuesta de LeBlanc (2006) sobre la asimetría como factor determinante en la guerra. 


\section{Presencia/ausencia de zonas de amortiguamiento}

Los cronistas describen que, en los llanos, las concentraciones de población estaban generalmente separadas entre sí por áreas extensas libres de ocupación humana, las cuales podrían interpretarse como zonas de amortiguamiento, que frecuentemente son asumidas como indicadores de guerra. Sin embargo, estas áreas libres de asentamientos, por sí solas, no son evidencia de zonas de amortiguamiento, ya que se requiere la existencia de algunas condiciones para que lo sean. Por ejemplo, en regiones donde la agricultura es la base de la subsistencia y de la economía política, las zonas de amortiguamiento son buena evidencia de guerra si la tierra cultivable es escasa y esas áreas vacías incluyen amplias extensiones de suelos productivos que deberían ser usados (Arkush, comunicación personal; LeBlanc 2006). La guerra y el crecimiento poblacional en una región con zonas de amortiguamiento extensas tienen como efecto una reducción significativa en la capacidad de carga de una población que está alcanzando sus límites en cuanto a la utilización de recursos, lo que causa presión sobre los recursos (LeBlanc 2006). Morey (1975) ha planteado que la falta de buena tierra para cultivo fue el principal motivo para la guerra en los llanos. De haber sido así, se espera que la tierra agrícola haya sido escasa en la región y que las zonas de amortiguamiento en los llanos hayan incluido importantes extensiones de dichos suelos. En consecuencia, la presencia de estas áreas de tierras agrícolas sin utilizar pudo generar presión poblacional sobre los recursos, tal como lo prevé el modelo de LeBlanc (2006).

Para observar la presencia de zonas de amortiguamiento en Barinas utilicé el análisis de densidad de Kernel, el cual permite generar mapas de densidad de puntos en 2D y ver las posibles agrupaciones que ellos conforman. Este tipo de análisis es alternativo a los histogramas y provee una representación eficiente de los datos (Baxter, Beardah y Wright 1997). Para el análisis de densidad de Kernel he usado el programa PAST. En estos análisis he utilizado los mismos parámetros para definir los límites de las unidades políticas que en los análisis de centralización demográfica.

En relación con la región de Gaván, usando la distancia radial de 12 kilómetros, el análisis de densidad de Kernel muestra cuatro agrupaciones de sitios bien definidas en el interior de dicha región (figura 1). Esta tendencia de la población a nuclearse dentro de la región de Gaván podría interpretarse como un rasgo defensivo ante posibles ataques que provinieran desde el interior o el exterior de la unidad política. 
Figura 1. Densidad de Kernel en la región de Gaván. Periodo Gaván Tardío

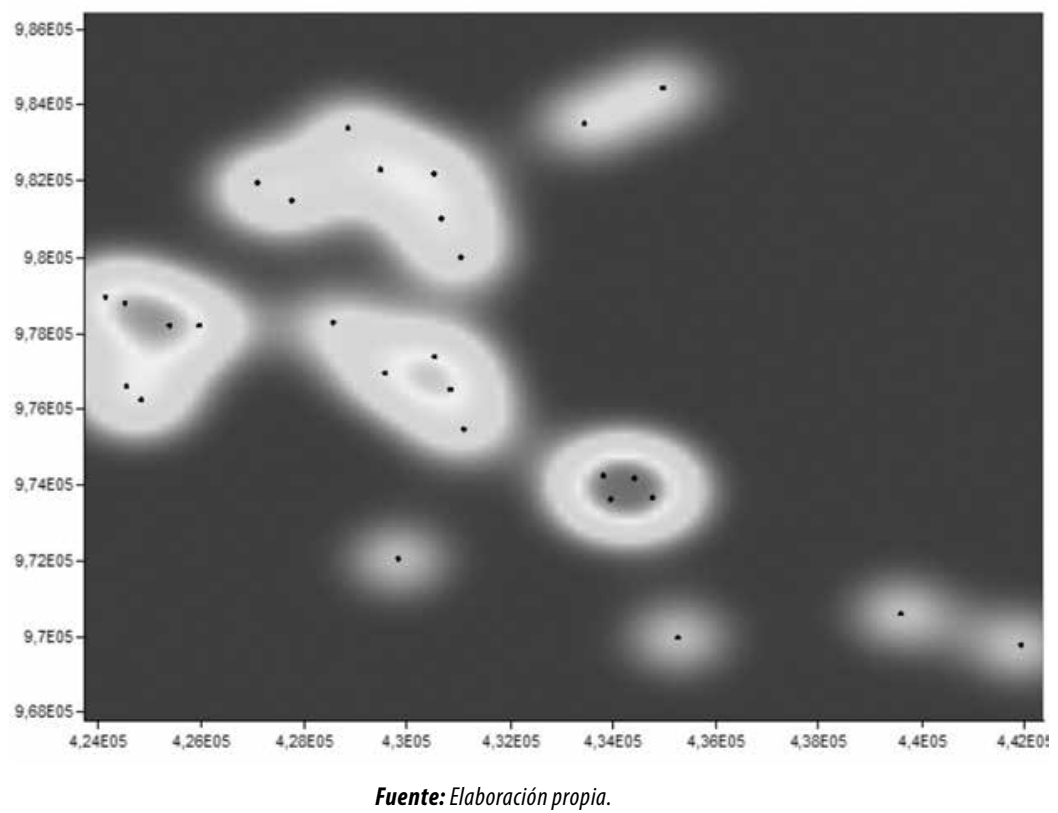

En el caso de la región del Cedral, cuando tomamos solo los sitios incluidos en los límites de $12 \mathrm{~km}$, el análisis muestra una clara y definida concentración de asentamientos alrededor del centro primario regional (figura 2).

Figura 2. Densidad de Kernel en la región de Cedral. Distancia radial 12 kilómetros.

Periodo Gaván Tardío

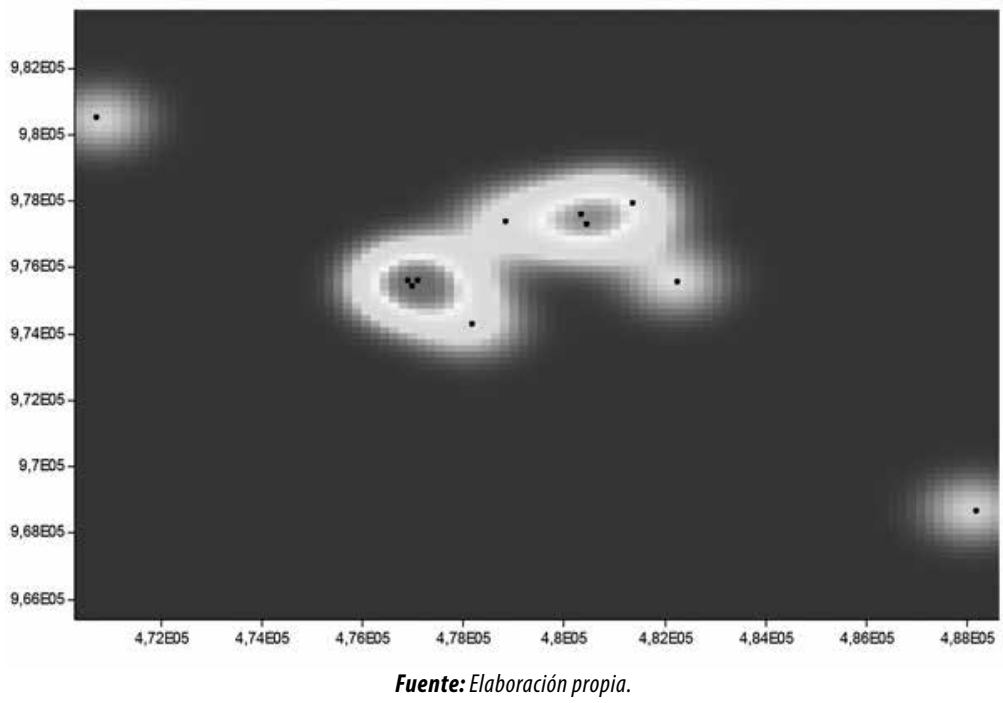


Figura 3. Densidad de Kernel. Región el Cedral. Periodo Gaván Tardío. Incluye todos los sitios conectados por calzadas

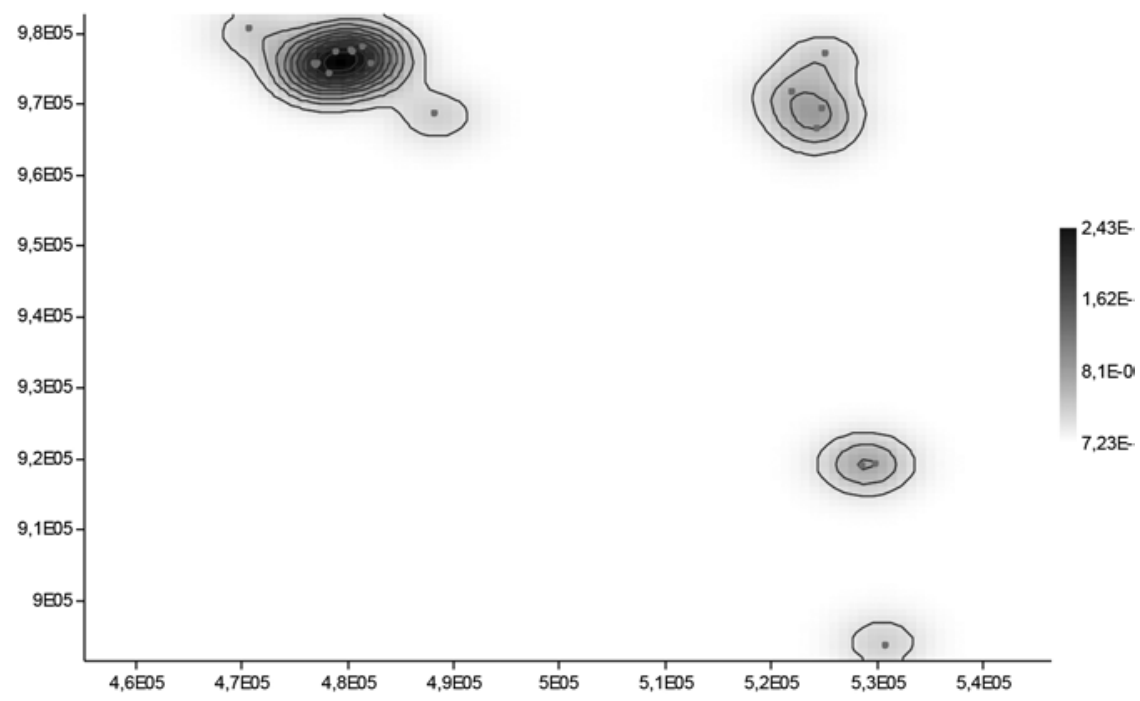

Fuente: Elaboración propia.

$\mathrm{Al}$ incluir todos los sitios unidos al centro regional por calzadas, se observan cuatro concentraciones claramente definidas, separadas entre sí por zonas vacías (figura 3). Es evidente la existencia de zonas libres de ocupación entre las distintas agrupaciones de asentamientos ubicadas en la unidad política del Cedral.

Como indiqué anteriormente, una de las características de la guerra es que es un fenómeno que puede manifestarse en forma distinta en diferentes escalas. Por esta razón, decidí realizar el análisis, pero esta vez incluyendo todos los asentamientos de las regiones de Gaván y Cedral. El análisis de densidad de Kernel nos muestra cuatro claras concentraciones de sitios separadas entre sí a escala macrorregional (figura 4).

En términos regionales, es posible afirmar que, en general, durante el periodo Gaván Tardío, la población tendió a concentrarse, lo que produjo la conformación de agrupaciones dentro de cada unidad política (figura 5).

A pesar de que estas agrupaciones se encontraban separadas entre sí por zonas vacías, estaban conectadas por la red de calzadas dentro de cada unidad política, lo que sugiere que esta distribución no obedecía a un conflicto interno. Sin embargo, es posible que, debido a que no contamos con información suficiente sobre el periodo Gaván Temprano, lo que estemos viendo sea el resultado de 
Figura 4. Densidad de Kernel. Regiones de Gaván y Cedral. Periodo Gaván Tardío

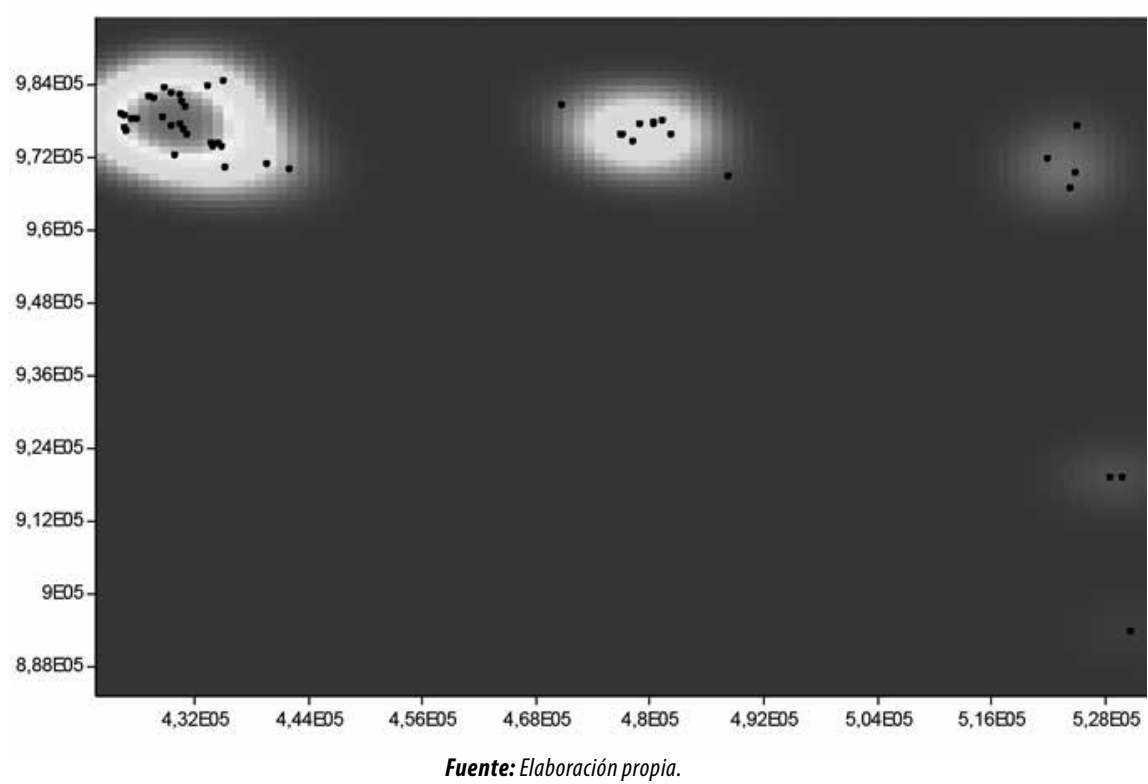

Figura 5. Posibles zonas de amortiguamiento entre las unidades políticas de Gaván y Cedral

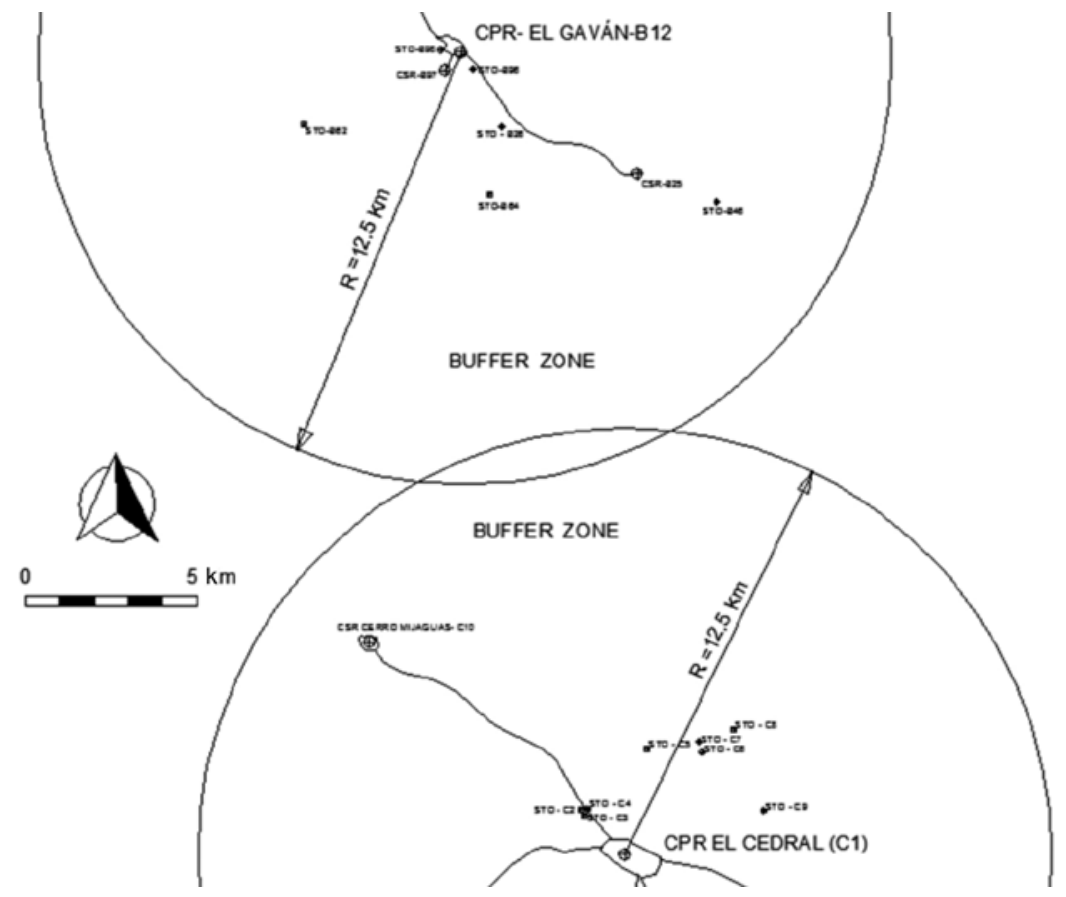

Fuente: Elaboración propia. 
un palimpsesto y, por tanto, lo que tengamos sea el final de un proceso de consolidación de unidades políticas que absorbieron a las comunidades locales en su respectiva región, lo que estaría de acuerdo con la hipótesis de la expansión territorial del modelo etnohistórico (Morey 1975; Morey y Marwitt 1975). Gassón ha propuesto, en este sentido, que

la presencia de zonas de amortiguamiento en las regiones de Cedral y Lomitas [...] podría sugerir una evolución autónoma de dichas unidades hasta que una se anexó o se alió a la otra. El análisis preliminar de la cerámica de los centros de Cedral y Lomitas indica la existencia de dos fases: una en la que cada uno de ellos poseía un estilo específico, y luego una segunda en la que surge un estilo único. Todo lo anterior sugiere un proceso de fusión de Lomitas con su vecino El Cedral. (2006, 42-43)

La existencia de agrupaciones de asentamientos separadas entre sí por “tierras de nadie” no es condición suficiente para definir estas áreas vacías como zonas de amortiguamiento. Como mencioné anteriormente, se requiere, por una parte, que estas zonas vacías correspondan a suelos fértiles en regiones donde hay escasez de tierras para cultivo y, por otra, que haya existido presión poblacional sobre los recursos. La primera de estas condiciones no se cumple, en cuanto los suelos que hacen parte de estas zonas vacías son de bajo potencial para uso agrícola. En relación con la segunda condición, los análisis sobre distribución de suelos indican que, en la región, hubo suficientes suelos aptos para la agricultura, para alimentar a toda la población de ambas regiones (Vargas 2012).

Por otra parte, las proyecciones demográficas realizadas han demostrado que ni en la región de Gaván ni en la de Cedral la población excedió el potencial productivo y que, al contrario, la agricultura permitió la generación de excedentes en cantidad considerable (Gassón 1998; Spencer, Redmond y Rinaldi 1994; Vargas 2012). Es decir, al parecer, en los llanos de Barinas la configuración de zonas de amortiguamiento no corresponde a la competencia por suelos aptos para el cultivo, como sugiere el modelo etnohistórico, y quizá otros factores, como la competencia por mano de obra, hayan sido la causa de esta configuración en la distribución de la población. Desde el punto de vista de Morey y de Morey y Marwitt, la captura de mano de obra y la captura de tierra son causas económicas, por lo que su modelo solo sería invalidado parcialmente. Finalmente, las dos condiciones que predice el modelo de LeBlanc (2006), presión poblacional y escasez de tierras para cultivo, que determinan la configuración de zonas de amortiguamiento, no se cumplirían en Barinas. Según estos resultados, si bien la guerra pudo estar presente en Barinas, la nucleación de población y la configuración de zonas 
libres de asentamientos no habrían sido el resultado de un conflicto intrarregional, y otros factores de tipo demográfico, ambiental o productivo pudieron haber impulsado la agregación de población.

\section{Organización agrícola}

Stone (1993) ha argumentado que dos componentes claves en la conformación de los patrones de asentamiento son la interacción entre las áreas de cultivo y la distancia entre los asentamientos en cualquier región, las cuales están muy relacionadas con el aprovisionamiento de fuerza de trabajo agrícola. Este investigador también ha propuesto que la atracción generada por la intensificación en el uso de la tierra, al momento de seleccionar dónde ubicar las unidades domésticas, es un principio de la organización espacial humana (Stone 1993).

Los cacicazgos de Moundville podrían ser un buen ejemplo del fenómeno descrito por Stone. En el valle Black Warrior, Myer (2002) ha hecho un reconocimiento sistemático en dos sectores, y observó que la distribución de las unidades domésticas fue influida por la proximidad a los cursos de los ríos, a las construcciones monticulares y a otros asentamientos rurales. Myer plantea que la continuidad en el uso de la tierra, entre el periodo West Jefferson (A. D. 900-1050) y el periodo Moundville I (A. D. 1050-1250), sugiere que la localización de los sitios monticulares secundarios no influyó en la distribución de los asentamientos rurales, sino que fue un proceso espacial que sucedió a la inversa. Según Myer, existe la posibilidad de que los montículos y su élite asociada hayan sido localizados cerca o en medio de una agrupación preexistente de sitios agrícolas, en vez de que los sitios agrícolas hubieran sido ubicados cerca de un montículo preexistente. De ser así, entonces, las tendencias de agregación pudieron generarse inicialmente por la disposición de la fuerza de trabajo en relación con la tierra para cultivo y no por la presencia de sitios monumentales.

En los llanos de Barinas es posible observar que, en la región de Gaván, existen agrupaciones de asentamientos alrededor de áreas de campos elevados que coinciden con áreas con suelos productivos, en las que fueron erigidas construcciones monticulares (Vargas 2011). En la región de Cedral, la mayor parte de la población se distribuyó en un sitio monumental construido en un banco aluvial que incluye los peores suelos agrícolas, mientras los mejores suelos para cultivo ubicados en la periferia permanecieron relativamente libres de ocupación. Estas áreas para cultivo fueron adecuadas con campos elevados, en los cuales han sido registrados sitios pequeños que corresponden posiblemente a 
unidades domésticas de familias dedicadas a la agricultura (Redmond, Gassón y Spencer 1999; Vargas 2012). Adicionalmente, hay algunas zonas pertenecientes a la unidad política de Cedral, donde existen centros secundarios con arquitectura monumental, en los que no hay presencia de estructuras defensivas, pero sí se registran extensas zonas de campos de cultivo desde las que parten calzadas hacia el centro regional. Esta información sugiere que es posible que los habitantes prehispánicos de los llanos hayan optado por localizar asentamientos monumentales alrededor de amplias zonas para cultivo, que pudieron ser trabajadas colectivamente por distintas comunidades. Las características de los patrones de asentamiento indican que la distribución espacial de los asentamientos en las regiones de Gaván y Cedral, durante el periodo Gaván Tardío, posiblemente se relacionó más con factores agrícolas que bélicos, algo muy similar a lo observado en el valle Black Warrior. Dichos factores tal vez fueron la necesidad de asegurar el fácil acceso a suficiente cantidad de suelos fértiles, y la coordinación y movilización de fuerza de trabajo. Estas condiciones habrían surgido como consecuencia de la intensificación de la producción agrícola llevada a cabo por los habitantes prehispánicos de Barinas, que requirió la inversión de una cantidad mucho mayor de energía por unidad de tierra, como plantea Stone (1993). Dicha estrategia quizá permitió disponer de suficiente fuerza de trabajo y, de forma secundaria, coordinar la defensa de los sitios ante posibles incursiones enemigas.

Como vemos, algunos de los patrones de asentamiento en los llanos de Barinas sugieren que la agricultura fue un elemento que afectó en forma importante la distribución de la población. Drennan y Peterson (2005) han señalado al respecto que la organización de la producción agrícola es un aspecto central relacionado con la configuración del tamaño de los asentamientos y su composición.

En consecuencia, pareciera ser que, tal como lo plantearon los cronistas, las poblaciones prehispánicas ubicaron sus asentamientos teniendo en cuenta que sus localizaciones fueran favorables para la agricultura. Sin embargo, no se puede simplificar este planteamiento, pues, como vimos en el caso de las regiones de Gaván y Cedral, posiblemente hubo distintas estrategias para la producción de alimentos. Mientras gran parte de la población de Gaván se asentó en los mejores suelos, en contraste, la mayor parte de los habitantes de la región del Cedral que residió en el centro regional ocupó tierras con bajo potencial productivo, y dejó disponibles las mejores para cultivo. Lo anterior está acorde con las crónicas, las cuales señalan que los indígenas de los llanos ubicaron sus asentamientos en relación con los campos de cultivo (Morey 1975; Rivero 1956).

En resumen, la información recolectada sugiere que la organización de la agricultura fue el elemento clave que generó las primeras tendencias a la 
centralización demográfica en los llanos de Barinas, posiblemente hacia finales del periodo Gaván Temprano. Un problema para observar este fenómeno es que los periodos cronológicos que han sido definidos para los llanos son demasiado amplios (más de cuatrocientos años), lo que impide observar qué sucedió entre los periodos Temprano y Tardío. En mi opinión, la guerra pudo ser un fenómeno que se presentó ya avanzado el periodo Gaván Tardío, en la medida en que los requerimientos de mano de obra para trabajar en los campos de cultivo aumentaron al intensificarse la producción.

\section{Defendibilidad de los asentamientos}

Las descripciones europeas mencionan la existencia de poblados fortificados. Estructuras para la defensa, como empalizadas, muros y zanjas, han sido asociadas en otras regiones del mundo con la guerra (Allen 2008; Arkush 2009; Champion 1982; Dye 2006; Spencer y Redmond 1998). La magnitud de estas estructuras y su distribución diferencial entre asentamientos son indicadores de la naturaleza e intensidad del conflicto y la violencia en una región. He reseñado anteriormente que los centros primarios de las regiones de Gaván y Cedral, según las investigaciones arqueológicas, fueron poblados fortificados similares a los descritos por los cronistas europeos. Uno de los rasgos más importantes en Barinas es la ausencia de estructuras defensivas en la mayoría de los centros secundarios, de los cuales la excepción es el sitio de Lomitas Florideñas (Gassón 1998; Vargas 2011).

Esta particularidad de los patrones de asentamiento sirve para evaluar la magnitud, la frecuencia y las características del conflicto en Barinas. Por ejemplo, en una región en la cual todos los asentamientos estén protegidos con estructuras de magnitud considerable es muy posible que la guerra sea endémica. En regiones donde los lugares centrales tienen estructuras defensivas, es muy probable que la confrontación involucre grupos de élite de distintas unidades políticas. También es importante considerar el componente ritual de la guerra, pues es probable que los sitios más antiguos, que eran no solo los centros políticos sino también los centros ceremoniales, hayan sido los principales objetivos de ataque. Esto podría explicar por qué el sitio El Gaván estaba encerrado y fue atacado, si la propuesta de Redmond, Gassón y Spencer (1999) es correcta.

De esta forma, se tiene que, a juzgar por la magnitud y localización de las estructuras defensivas en los sitios, la intensidad de la confrontación en los llanos de Barinas no fue endémica y quizá haya involucrado prioritariamente a las élites regionales y sus cohortes y haya tenido un componente ritual, como ha sido reportado con respecto a otras regiones en el Orinoco (Whitehead 1994). 


\section{Conclusiones}

Los modelos arqueológicos y etnohistóricos propuestos para estudiar el surgimiento de cacicazgos en los llanos de Colombia y Venezuela se han concentrado en la guerra como una de las principales causas del surgimiento de organización social compleja en la región. En este artículo he presentado una propuesta para analizar este fenómeno que incluye la evaluación de algunas variables, como la agregación de la población, la centralización demográfica, el tamaño de las unidades políticas, la presencia/ausencia de zonas de amortiguamiento, la organización agrícola y la defendibilidad de los sitios.

Los análisis presentados anteriormente sugieren como hipótesis que la organización de la agricultura fue el elemento clave que generó las primeras tendencias a la agregación y la centralización demográfica en los llanos de Barinas y no la guerra, como ha sugerido Spencer (1998). Esta pudo ser un fenómeno que se presentó posteriormente, en la medida en que los requerimientos de mano de obra aumentaron al intensificarse la producción agrícola. Los datos disponibles sugieren que la guerra en los llanos no fue un resultado de presión poblacional ni de escasez de tierras aptas para el cultivo, como propone el modelo etnohistórico y ecológico de Morey (1975) y Morey y Marwitt (1975). Estos mismos resultados indican que, en Barinas, no son estas dos variables las que determinan la configuración de zonas de amortiguamiento, como postula el modelo de LeBlanc (2006). Es posible que, como argumentan Whitehead (1994), Drennan (1995) y Gassón (1998), la escasez de mano de obra necesaria para intensificar la agricultura y expandir la frontera agrícola y política haya sido la causa de la guerra en Barinas y quizá en otras partes de los llanos del Orinoco. Desde esta perspectiva, la configuración de zonas de amortiguamiento también podría haberse dado como resultado de la competencia por fuerza de trabajo. Finalmente, se plantea que la guerra en los llanos de Barinas no fue un fenómeno endémico y que es muy posible que haya tenido un fuerte componente ritual que involucró principalmente a los grupos de élite.

La guerra, como hemos visto, supone un rango de variabilidad no solamente relacionado con el liderazgo, el prestigio personal y el estatus, sino también con sus causas, su frecuencia, su intensidad, su escala espacial y temporal. Esta variabilidad pudo haber generado distintas trayectorias de cambio que resultaron en diversas formas de organización social, que al parecer existieron en el Orinoco antes de la llegada de los invasores europeos. Será importante adelantar estudios similares en los llanos de Colombia para establecer cuáles son las diferencias y semejanzas con la trayectoria evolutiva de las sociedades llaneras de Venezuela. 
La evaluación arqueológica de la guerra en los llanos de Colombia y Venezuela requerirá analizar cómo los patrones de asentamiento cambiaron a través del tiempo. Para lograr este objetivo en los llanos del Orinoco, es necesario refinar los esquemas cronológicos existentes para poder entender mejor cómo estos cambios espaciales estuvieron asociados a transformaciones en la organización social de los grupos llaneros. Por ejemplo, la construcción de empalizadas y encerramientos, la nucleación de población, el aumento en las tendencias a la centralización, la configuración de zonas de amortiguamiento y el incremento en los niveles de conflicto y violencia intrarregional pueden corresponder a periodos de fisión de asentamientos producida por procesos de fragmentación política. En el otro extremo, el incremento demográfico continuo, la eliminación de zonas de amortiguamiento, la intensificación agrícola en campos elevados, la construcción de calzadas y caminos ubicados en áreas ecológicas a las que tenían acceso distintos asentamientos indicarían la fusión de comunidades en unidades políticas más amplias, producto de la reducción en el conflicto intrarregional. Es probable que la consolidación de una autoridad regional coincida con la aparición de nuevas formas de conflicto y violencia; también, con nuevos sistemas de producción agroecológica.

Aunque las fuentes etnohistóricas y los modelos arqueológicos han hecho énfasis en la importancia de la guerra para las poblaciones prehispánicas en el Área Intermedia, será necesario asumir esta proposición como una hipótesis más que como una conclusión. El estudio comparativo de la guerra ofrece una oportunidad de entender el papel que este fenómeno desempeñó en el surgimiento de nuevas formas de organización social en el Orinoco y otras regiones del Área Intermedia.

\section{Agradecimientos}

Para el presente artículo han sido fundamentales la guía y las sugerencias de los profesores Elizabeth Arkush y Robert Drennan, ambos docentes de la Universidad de Pittsburgh. También agradezco al doctor Rafael Gassón, ya que algunas de las ideas que presento en este artículo fueron abordadas y discutidas embrionariamente en mi tesis de maestría, la cual desarrollé bajo su dirección durante mi paso por el Laboratorio de Ecología Humana del Instituto Venezolano de Investigaciones Científicas (IVIC). 


\section{Referencias}

Aguado, Pedro. 1950. Historia de Venezuela. Vol. 1. Madrid: Maestre.

Allen, Mark. 2006. "Transformations in Maori Warfare: Toa, Pa, and Pu”. En Arkush y Allen 2006, 184-213.

-. 2008. "Hillforts and the Cycling of Maori Chiefdoms: Do Good Fences Make Good Neighbors? En Global Perspectives on the Collapse of Complex Systems, editado por J. A. Railey y R. M. Reycraft, 65-81. New Mexico, Albuquerque: Maxwell Museum of Anthropology.

Anderson, David G. 1994. The Savannah River Chiefdoms: Political Change in the Late Prehistoric Southeast. Alabama: The University of Alabama Press.

Arkush, Elizabeth. 2009. "Warfare, Space, and Identity in the South-central Andes: Constraints and Choices”. En Warfare in Cultural Context: Practice, Agency, and the Archaeology of Violence, editado por A. E. Nielsen y W. Walker, 190-217. Tucson: University of Arizona Press.

—. 2010. Comunicación personal. Octubre.

Arkush, Elizabeth y Mark Allen, eds. 2006. The Archaeology of Warfare: Prehistories of Raiding and Conquest. Gainesville: University of Florida Press.

Arkush, Elizabeth y Charles Stanish. 2005. "Interpreting Conflict for the Archaeology of Warfare". Current Anthropology 46: 3-28.

Baxter, M., C. Beardah y R. Wright. 1997. "Some Archaeological Applications of Kernel Density Estimates”. Journal of Archaeological Science 24: 347-354.

Carvajal, Jacinto de. 1985. Descubrimiento del río Apure. Madrid: Historia 16.

Castellanos, Juan. 1962. Elegías de varones ilustres de Indias. Biblioteca de la
Academia Nacional de la Historia 57. Caracas: Italgráfica.

Champion, Timothy. 1982. "Fortification, Ranking and Subsistence”. En Ranking, Resource and Exchange: Aspects of the Archaeology of Early European Society, editado por C. Renfrew y S. Shennan, 61-66. Cambridge: Cambridge University Press.

Drennan, Robert. 1995. "Chiefdoms in Northern South America”. Journal of World Prehistory 9 (3): 301-340.

\section{Drennan, Robert y Christian Peterson.}

2008. "Centralized Communities, Population, and Social Complexity after Sedentarization". En The Neolithic Demographic Transition and its Consequences, editado por Jean-Pierre Bouquet-Appel y Ofer Bar-Yosef, 359-386. Nueva York: Springer.

Dye, David. 2006. “The Transformation of Mississippian Warfare. Four Case Studies from the Mid-South”. En Arkush y Allen 2006, 101-147.

Earle, Timothy. 1997. How Chiefs Come to Power: The Political Economy in Prehistory. Stanford: Stanford University Press.

Federmann, Nicolás. 1958. Historia indiana. Madrid: ARO.

Ferguson, Brian. 1990. "Explaining War". En The Anthropology of Warfare, editado por J. Haas, 26-55. Cambridge: Cambridge University Press.

Gassón, Rafael. 1998. "Prehispanic Intensive Agriculture, Settlement Pattern and Political Economy in the Western Venezuelan Llanos”. Tesis de doctorado, University of Pittsburgh, Department of Anthropology, Pittsburgh.

-. 2006. "Los sabios ciegos y el elefante: sistemas de intercambio y organizaciones sociopolíticas en el Orinoco y áreas 
vecinas en la época prehispánica”. En Contra la tiranía tipológica en arqueología: una visión desde Suramérica, editado por Cristóbal Gnecco y Carl Langebaek, 31-54. Bogotá: Universidad de los Andes; CESO.

—. 2009. "Apolo y Dionisos en el occidente de Venezuela: antiguas sociedades complejas de los llanos de Barinas”. En Economía, prestigio y poder. Perspectivas desde la arqueología, editado por Carlos Augusto Sánchez, 17-41. Bogotá: Instituto Colombiano de Antropología e Historia.

Gassón, Rafael y Juan Carlos Rey. 2006. "Cacicazgos cíclicos e intensificación agrícola en los llanos occidentales de Venezuela”. En Agricultura ancestral: camellones y albarradas, editado por Francisco Valdez, 141-158. Quito: Abya-Yala.

Gassón, Rafael y Alberta Zucchi. 2010. “El cambio climático y la diáspora arawaka: algunas correlaciones entre el cambio sociocultural y ambiental en Venezuela prehispánica”. Ponencia presentada en la V Reunión de Teoría Arqueológica de América del Sur, Caracas, Venezuela.

Gumilla, Joseph. 1963. El Orinoco ilustrado y defendido. Caracas: Academia Nacional de la Historia.

Heckenberger, Michael. 2002. "Rethinking the Arawakan Diaspora: Hierarchy, Regionality and the Amazonian Formative”. En Comparative Arawakan Histories: Rethinking Language Family and Culture Area in Amazonia, editado por J. Hill y F. Santos-Granero, 99-122. Urbana y Chicago: University of Illinois Press.

Junker, Laura. 1999. Raiding, Trading, and Feasting: The Political Economy of Philippine Chiefdoms. Honolulu: University of Hawai'i Press.

Keeley, Lawrence H., Marisa Fontana y Russell Quick. 2007. "Battles and Bastions: The Universal Features of
Fortifications". Journal of Archaeological Research 15: 55-95.

Kintigh, Keith. 1990. "Intrasite Spatial Analysis: A Commentary on Major Methods”. En Mathematics and Information Science in Archaeology: A Flexible Framework, editado por Albertus Voorrips, 165-200. Bonn: Holos.

Kolb, Michael y Boyd Dixon. 2002. "Landscapes of War: Rules and Conventions of Conflict in Ancient Hawai'i (and Elsewhere)". American Antiquity 67: 514-534.

Lau, George. 2010. "Fortifications as Warfare Culture: The Hilltop Centre of Yayno (Ancash, Peru), AD 400-800”. Cambridge Archaeological Journal 20 (3): 419-448.

LeBlanc, Stephen. 2006. "Warfare and the Development of Social Complexity”. En Arkush y Allen 2006, 437-468.

Morey, Nancy. 1975. "Etnohistory of the Colombian and Venezuelan Llanos”. Tesis de doctorado, Department of Anthropology, University of Utah, Provo.

Morey, Robert y John Marwitt. 1975. "Ecology, Economy, and Warfare in Lowland South America”. En World Anthropology: Advances in Andean Archaeology, editado por W. de Druyter, 247-258. Chicago: Mouton.

Myer, Jennifer. 2002. "Characteristics of Mississippian Settlement in the Black Warrior Valley: Final Report of Season III of the Black Warrior Valley Survey". Alabama Historical Commission, Black Warrior Survey.

Peterson, Christian E. y Robert D. Drennan. 2005. "Communities, Settlements, Sites and Surveys: Scale Analysis of Prehistoric Human Interaction”. American Antiquity 70: 5-30.

Pinder, D., I. Shimada y D. Gregory. 1979. "The Nearest-Neighbor Statistic: 
Archaeological Application and New Developments”. American Antiquity 44: 430-445.

\section{Redmond, Elsa, Rafael Gassón y Charles} Spencer. 1999. "A Macroregional View of Cycling Chiefdoms in the Western Venezuelan Llanos". En Complex Polities in the Ancient Tropical World, editado por E. Bacus y L. Lucero, 109-130. Archaeological Papers of the American Anthropological Association 9. Arlington: American Anthropological Association.

Rivero, Juan. 1956. Historia de las misiones de los llanos de Casanare y los ríos Orinoco y Meta. Bogotá: Argra.

Roscoe, Paul. 2008. "Settlement Fortification in Village and 'Tribal' Society: Evidence from Contact-Era New Guinea”. Journal of Anthropological Archaeology 27: 508-519.

Spencer, Charles. 1993. "Human Agency, Biased Transmission, and the Cultural Evolution of Chiefly Authority”. Journal of Anthropological Archaeology 12: 41-74.

-. 1994. "Factional Ascendance, Dimensions of Leadership, and the Development of Centralized Authority”. En Factional Competition and Political Development in the New World, editado por Elizabeth M. Brumfiel y John W. Fox, 31-43. Cambridge: Cambridge University Press.

-. 1998. "Investigating the Development of Venezuelan Chiefdoms”. En Chiefdoms and Chieftaincy in the Americas, editado por Elsa M. Redmond, 104-137. Gainesville: University of Florida Press.

Spencer, Charles y Elsa Redmond. 1992. "Prehispanic Chiefdoms of the Western Venezuelan Llanos”. World Archaeology 24: 134-157.
-. 1998. "Prehispanic Causeways and Regional Politics in the Llanos of Barinas, Venezuela”. Latin American Antiquity 9 (2): 95-110.

Spencer, Charles, Elsa Redmond y Milagro Rinaldi. 1994. "Drained Fields at La Tigra, Venezuelan Llanos: A Regional Perspective”. Latin American Antiquity 5 (2): $119-143$

Stone, Glenn. 1993. "Agrarian Settlement and the Spatial Disposition of Labor". En Spatial Boundaries and Social Dynamics: Case Studies from Food-Producing Societies, vol. 2, editado por A. Holl y T. E. Levy, 25-38. Ann Arbor: International Monographs in Prehistory.

Whitehead, Neil. 1994. "The Ancient Amerindian Polities of the Amazon, the Orinoco, and the Atlantic Coast: A Preliminary Analysis of their Passage from Antiquity to Extinction”. En Amazonian Indians: From Prehistory to the Present, editado por Anna Roosevelt, 33-54. Tucson: University of Arizona Press.

-. 1998. "Colonial Chieftains of the Lower Orinoco and Guayana Coast”. En Chiefdoms and Chieftaincy in the Americas, editado por Elsa M. Redmond, 150-163. Gainesville: University Press of Florida.

Vargas, Juan Carlos. 2011. "Patrones de asentamiento y distribución de recursos agrícolas en dos unidades políticas prehispánicas en los llanos occidentales de Venezuela”. Tesis de maestría, Instituto Venezolano de Investigaciones Científicas (IVIC), Caracas.

-. 2012. "Patrones de asentamiento y distribución de recursos agrícolas en dos unidades políticas prehispánicas en los llanos occidentales de Venezuela”. Maguaré 26 (1): 195-228. 